\title{
Comparative Analysis of Serum Cytokine ELISA and Multiplex Techniques
}

\author{
Corey T Ungaro ${ }^{1 *}$, Anthony S Wolfe ${ }^{2}$ and Shyretha D Brown ${ }^{1}$ \\ ${ }^{1}$ Gatorade Sports Science Institute, PepsiCo R\&D Life Sciences, Barrington IL, USA \\ ${ }^{2}$ Gatorade Sports Science Institute, PepsiCo R\&D Life Sciences, Frisco TX, USA
}

*Corresponding author: Corey T Ungaro, Gatorade Sports Science Institute, PepsiCo R\&D Life Sciences, Barrington IL, USA

\section{ARTICLE INFO}

Received: 蔧 December 17, 2020

Published: 幽 December 23, 2020

Citation: Corey T Ungaro. Comparative Analysis of Serum Cytokine ELISA and Multiplex Techniques. Biomed J Sci \& Tech Res 32(5)-2020. BJSTR. MS.ID.005313.

\begin{abstract}
Enzyme-linked immunosorbent assay (ELISA) has been described as the gold standard for serum cytokine analysis. However, multiplex array technologies are increasing in popularity, likely because of their ability to analyze large numbers of analytes with low sample volume compared to ELISA. Few studies have directly compared serum cytokine results from ELISA and Multiplex analytical techniques. Therefore, the purpose of this observational research was to determine if differences exist in serum cytokine concentrations between ELISA and Multiplex techniques. Blood samples were collected from the antecubital vein of professional American football players during a competitive season to monitor biomarkers fluctuations throughout the season. After clotting, blood was centrifuged and three $300 \mu \mathrm{L}$ aliquots of serum were frozen at $-80^{\circ} \mathrm{C}$. IL- $1 \beta$, IL-6, and TNF- $\alpha$ were measured from the exact same serum samples using ELISA (EMD Millipore, Sigma Aldrich) and Multiplex (EMD Millipore, Magpix) kits.
\end{abstract}

Coefficients of variation (CV\%), paired samples t-test with 95\% CI, and Pearson's product-moment correlations were used to compare cytokine analysis techniques. Descriptive statistics are shown as mean \pm SD. Results indicated that serum cytokine concentrations were not comparable between ELISA and Multiplex analytical techniques. IL-1 $\beta(n=15)$ displayed significant variability between techniques with a CV\% of 119.9 with ELISA reading $36.8 \pm 32.4 \mathrm{pg} / \mathrm{mL}$, intra-assay CV\%= 7.6 and Multiplex showing 1.1 $\pm 1.2 \mathrm{pg} / \mathrm{mL}$, intra-assay CV\%= 6.8. IL-6 $(\mathrm{n}=33)$ demonstrated substantial variability between techniques with a CV\% of 126.9 with ELISA reading $340.2 \pm 460.5 \mathrm{pg} / \mathrm{mL}$, intraassay CV\%= 15.3 and Multiplex showing $11.8 \pm 20.2 \mathrm{pg} / \mathrm{mL}$, intra-assay CV\%= 4.8. For TNF- $\alpha(n=36)$ all ELISA samples were below the detection limit of 0.31 with the Multiplex detecting $8.8 \pm 3.2 \mathrm{pg} / \mathrm{mL}$, intra-assay CV\%= 7.9. Moreover, paired samples t-tests showed considerable mean differences between analysis techniques for IL-1 $\beta(-35.8 \mathrm{pg} / \mathrm{mL}$, $(95 \% \mathrm{CI},-51.6$ to -19.9$) \mathrm{t}(17)=-4.8, \mathrm{p}<0.001)$ and IL-6 $(-328.4 \mathrm{pg} / \mathrm{mL}(95 \% \mathrm{CI},-490.0$ to $-166.7), \mathrm{t}(32)=-4.1, \mathrm{p}<0.001$.

Pearson's correlation between ELISA and Multiplex was not significant for IL-1 $\beta$ $[\mathrm{r}(13)=0.44, \mathrm{p}=0.068]$ or IL- $6[\mathrm{r}(31)=0.25, \mathrm{p}=0.157]$. The results suggest that ELISA and Multiplex outcomes were not comparable and the differences in blocking agents used may be the source of erraticism. According to the results from this study, comparisons between analytical techniques should be avoided and more work is needed to determine the cause of these large discrepancies seen between methods.

\section{Introduction}

Inflammation is a protective response that is necessary for responding to invading pathogens. Cytokines, secreted by immune cells, play a major role in this response. Measurement and detection of cytokine levels in serum are essential in understanding biological homeostasis to maintain human health. Investigation of cytokines in serum analysis has been classically conducted by enzyme-linked immunosorbent assay (ELISA). With an increasing demand for rapid and precise measurements for multiple cytokines and other biomarkers, ELISA has been the standard for quantitative serum analysis. Yet, it is not well suited for high throughput multiple 
biomarker detection [5]. Compared to ELISA, multiplex arrays provide quantitative measurement of multiple cytokines on a largescale using bead-based technology. This sought-after assay offers a quantitative measure of multiple cytokines on a large-scale using bead-based technology. Thus, further analysis of these two methods for quantitative serum cytokine analyses is necessary.

ELISA has been classically described as the gold standard for serum cytokine analysis [14]. Also known as the sandwich ELISA, an antibody adhered to the bottom of a well provides capturing antigen capability and immune specificity, and a different antibody provides detection and amplification. The method described has been cited as an accurate and sensitive reading of the cytokine of interest [16-19]. However, the data reading outcome depends on the kit manufacturer, antibody quality, and skill and experience of the operator [14]. On the other hand, the ELISA method provides some limitations. For example, only one cytokine at a time can be measured, limiting the ability of investigators to analyze various cytokines of interest due to limited amount of biological sample available $[9,19]$. Moreover, ELISA limits the range of cytokine detection, meaning the linear relationship between cytokine concentration and absorbance reading is narrow compared to other technologies such as multiplex arrays.

ELISA samples above the cytokine range require an additional kit for re-testing with a dilution factor creating additional labor, cost, and valuable sample volume, if extra sample is available. Diluted ELISA values may not be comparable to undiluted ELISA values. This is applicable to serum samples when interpreting the differences in ELISA results between samples that show results within the cytokine range (not requiring dilution) and samples above the cytokine range (requiring dilution) [19]. Multiplex array technologies are increasing in popularity; this is likely due to their ability to simultaneously analyze large numbers of cytokines from a single sample with low volume compared to ELISA [9]. This technology is a flow cytometric (bead-based) assay that allows each bead to be coated with a specific capture antibody. Detection antibodies labeled with a fluorescence compound bind to the specific cytokine-capture antibody complex on each bead. As a result, many cytokines in a biological sample can be detected by the difference in bead fluorescence for nearly 100 biomarkers [19]

When compared to ELISA, multiplex arrays offer these advantages: a) high throughput multiplex analysis, b) reduced sample volume, c) time and cost efficiency, d) ability to evaluate the levels of one given inflammatory biomarker in the context of multiple others, e) ability to perform repeated measures of the same cytokine panels in the same subjects under the same experimental assay conditions, f) ability to reliably detect different proteins across a broad dynamic range of concentrations [19]. The technology of choice is under the discretion of the investigators and depends on the research objective and the biological sample of concern. The comparison between ELISA and Multiplex arrays are acceptable when using tissue culture supernatant samples; however, the comparability is less promising when using serum or plasma samples [13]. Multiplex array technologies are more sensitive than ELISA to alterations in circulating proteins and inhibitors present in serum or plasma, which can complicate levels detected during disease, aging, or inflammatory conditions associated with exercise/sport. IL-1 $\beta$, IL-6, and TNF- $\alpha$ were selected as the biomarkers of interest to assess the degree of inflammation and muscle damage of professional American football players throughout a season

These biomarkers have been evaluated and monitored in other sports to determine recovery and inflammation in athletes $[1,8,11,12]$. Few studies have directly compared serum cytokine results from ELISA and Multiplex analytical techniques, specifically within healthy professional American football players. Therefore, the purpose of this study was to determine differences in serum cytokine concentrations between ELISA and Multiplex techniques.

\section{Materials and Methods}

\section{Study Population and Samples}

Blood samples were collected in Becton Dickinson (Franklin Lakes, NJ) vacutainers, according to International Review Board (Sterling IRB, Atlanta, GA) approval and the subject's signed informed consent. Samples came from the antecubital vein of healthy professional American football players during a competitive season. After collection, the whole blood remained at room temperature for 30 minutes to allow samples to coagulate per the vacutainer instructions. After coagulation, the blood was centrifuged, and three $300 \mu \mathrm{L}$ aliquots of serum were frozen at $-80^{\circ} \mathrm{C}$. To standardize the process, one technician carried out the respective assay protocols on the same day, under the same laboratory conditions, using the exact same serum samples to compare their results across Multiplex and ELISA cytokine kits.

\section{Determination of Cytokines}

ELISA Assay: Human IL-1 $\beta$ and Human IL-6 were measured using a competitive ELISA (Millipore Sigma, Sigma Aldrich), an in vitro quantitative assay kit used to detect biological peptides or proteins based on the competitive enzymes immunoassay principle. TNF- $\alpha$ was measured with a high sensitivity amplification ELISA (Invitrogen, Thermo Fisher Scientific). All ELISA assays were performed according to the manufacturer's instructions. $100 \mu \mathrm{L}$ of serum, in duplicate, were added to the microplates and incubated for 2.5 hours and washed $4 \mathrm{x}$ with wash buffer for all ELISA assays. For IL-1 $\beta$ and IL-6, the microplates used were pre-coated with a target-specific antibody (anti-IgG). The antibody was competitively bound by a mixture of biotinylated peptide (provided with the kit) and the endogenous peptide in the serum samples and incubated for 1 hour and washed $4 \mathrm{x}$ with wash buffer. The biotinylated peptide then interacted with HRP-Streptavidin and incubated for 45 
minutes followed by a $4 \mathrm{x}$ wash. Finally, TMB Reagent was added to each well and incubated for $30 \mathrm{~min}$ which produced a colorimetric signal to be read on a spectrophotometer.

For TNF- $\alpha$, each plate was coated with an anti-human TNF- $\alpha$ polyclonal antibody adsorbed onto the microwells. To the microwells, biotin-conjugated anti-human TNF- $\alpha$ antibody was added to attach to any detectable human TNF- $\alpha$ captured by the first antibody. Following a 2-hour incubation, unbound biotinconjugated anti-human TNF- $\alpha$ antibody was removed with an automatic plate washer. After the unbound antibody was removed, Streptavidin-HRP was added and bound to the biotin-conjugated anti-human TNF- $\alpha$ antibody. Following an hour incubation, unbound Streptavidin-HRP was removed during the washing step, and amplification reagent I (Biotinyl-Tyramide) was added to the wells. After a 15-minute incubation, unbound amplification reagent I was removed through washing, and amplification reagent II (Streptavidin-HRP) was added. Following a 30-minute incubation, unbound amplification reagent II was removed by washing, and a substrate solution reactive with HRP, TMB substrate, was added and incubated for 20-minutes.

In the last step, a colored product formed in proportion to the amount of human TNF- $\alpha$ present in the serum or standard. For all ELISA assays, the reactions were terminated with the addition of acid. The sample absorbance was measured at $450 \mathrm{~nm}$ using a spectrophotometer; the signal intensity was directly proportional to the amount of biotinylated peptide-streptavidin-HRP and inversely proportional to the amount of target peptide in the samples or standards. A standard curve was prepared from seven standard dilutions (IL-6 $=1.37-1,000 \mathrm{pg} / \mathrm{mL}, \mathrm{IL}-1 \beta=0.48-100 \mathrm{pg} /$ $\mathrm{mL}$, and TNF- $\alpha=0.31-20 \mathrm{pg} / \mathrm{mL}$ ) and serum sample concentrations were determined using Biotek Cytation 3 Multi-Reader (Biotek, Winooski, VT) with Gen5 software.

Multiplex Assay: IL-1 $\beta$, IL-6, and TNF- $\alpha$ were simultaneously measured using magnetic bead-based immunoassay Multiplex for comparisons to ELISA techniques. The Multiplex assay was performed according to the manufacturer's instructions. $25 \mu \mathrm{L}$ of serum sample, in duplicate, were assayed. The Multiplex assay involved beads of defined spectral properties conjugated to proteinspecific capture antibodies and added with the samples (including standards of known protein concentration and control and test samples) into the wells of a microplate. The target proteins bound to the capture antibodies for an overnight incubation. After manual washing of the beads using a magnetic plate, protein-specific biotinylated detector antibodies were added and incubated with the beads for 1-hour. Then, excess biotinylated detector antibodies were removed, and a streptavidin-conjugated fluorescent protein, R-phycoerythrin (SAV-RPE), was added and incubated for 30 minutes. SAV-RPE bound to the biotinylated detector antibodies, forming a four-member, solid-phase sandwich.
After washing to remove unbound SAV-RPE, the beads were analyzed, and standard curves (IL-6 $=0.18-750 \mathrm{pg} / \mathrm{mL}$, IL-1 $\beta=0.49$ $2000 \mathrm{pg} / \mathrm{mL}$, and TNF- $\alpha=0.43-1750 \mathrm{pg} / \mathrm{mL}$ ) along with individual well concentrations were determined using the Magpix multiplex system with xPonent software (Luminex, Austin, TX).

\section{Statistics}

Coefficients of variation (CV\%), paired-samples t-test with 95\% CI, and Pearson's product-moment correlations were used to compare cytokine analysis techniques, similar to other studies investigating differences between competing methods [4,7]. Descriptive statistics are shown as mean \pm SD. Statistical analyses were performed using Minitab software (Minitab Inc., State College, $\mathrm{PA})$.

\section{Results}

Serum cytokine concentrations were not comparable between ELISA and Multiplex analytical techniques. ELISA kits did not include internal quality controls. All standard curves performed as expected for each assay $\left(\mathrm{r}^{2}>0.99\right)$. Descriptive statistical comparison between ELISA and Magpix multiplex for IL-1 $\beta$ $(n=15)$ displayed significant variability between techniques with a CV\% of 119.9 (Table 1). IL-6 $(n=33)$ demonstrated substantial variability between techniques with a CV\% of 126.9 (Table 2). For TNF- $\alpha(n=36)$ all ELISA samples were below the detection limit of $0.31 \mathrm{pg} / \mathrm{mL}$ with the Magpix Multiplex detecting $8.8 \pm 3.2 \mathrm{pg} /$ $\mathrm{mL}$ (intra-assay $\mathrm{CV} \%=7.9$ ). Due to limited sample availability not all serum samples were able to be performed across all kits. Additionally, due to no detection of TNF- $\alpha$ from the ELISA kit, no statistical comparison could be determined between ELSIA and Multiplex. Paired samples t-tests showed considerable mean differences between analytical techniques for IL-1 $\beta$ and IL-6 (Table $3)$. Pearson correlation coefficients between ELISA and Multiplex were not significant for IL-1 $\beta[r(13)=0.44, p=0.068]$ or IL-6 $[r(31)$ $=0.25, \mathrm{p}=0.157]$ (seen in Figures 1\&2).

Table 1: Comparison between ELISA and Magpix IL-1 $\beta$.

\begin{tabular}{|c|c|c|}
\hline Descriptive Statistics & $\begin{array}{c}\text { ELISA IL-1 } \beta \\
\text { (pg/mL) }\end{array}$ & $\begin{array}{c}\text { Magpix IL-1 } \beta \text { (pg/ } \\
\mathbf{m L})\end{array}$ \\
\hline $\begin{array}{c}\text { Mean } \pm \text { SD (pg/mL) } \\
(\mathrm{n}=18)\end{array}$ & $1.1 \pm 1.2$ & $36.8 \pm 32.4$ \\
\hline $\begin{array}{c}\text { Intra-assay CV (\%) } \\
\text { Between Methods CV } \\
(\%)\end{array}$ & 6.8 & 7.6 \\
\hline
\end{tabular}

Table 2: Comparison between ELISA and Magpix IL-6.

\begin{tabular}{|c|c|c|}
\hline Descriptive Statistics & ELISA IL-6(pg/mL) & Magpix IL-6(pg/mL) \\
\hline $\begin{array}{c}\text { Mean } \pm \text { SD (pg/mL) } \\
(\mathrm{n}=33)\end{array}$ & $340.2 \pm 460.5$ & $11.8 \pm 20.2$ \\
\hline $\begin{array}{c}\text { Intra-assay CV (\%) } \\
\text { Between Methods CV } \\
(\%)\end{array}$ & 15.3 & 4.8 \\
\hline
\end{tabular}


Table 3: Paired Samples t-test between ELISA and Magpix IL-1 $\beta$ and IL-6.

\begin{tabular}{|c|}
\hline Paired Samples t-test \\
\hline $\mathrm{IL}-1 \beta(-35.8 \mathrm{pg} / \mathrm{mL},(95 \% \mathrm{CI},-51.6$ to -19.9$) \mathrm{t}(17)=-4.87 \mathrm{p}<0.001)$ \\
\hline $\mathrm{IL}-6(-328.4 \mathrm{pg} / \mathrm{mL}(95 \% \mathrm{CI},-490.0$ to -166.7$), \mathrm{t}(32)=-4.1, \mathrm{p}<0.001)$ \\
\hline
\end{tabular}

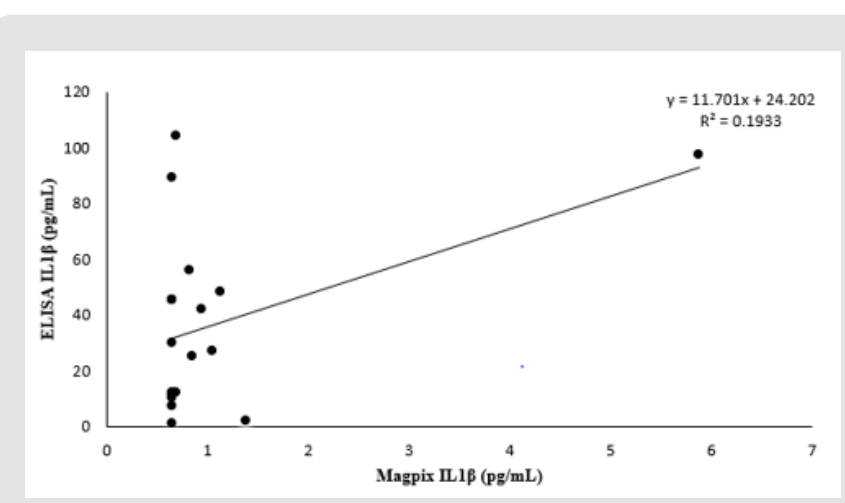

Figure 1: Method Comparison.

Note: Data expressed as ELISA IL-1 $\beta$ (pg/mL) vs. Magpix IL-1 $\beta$ ( $\mathrm{pg} / \mathrm{mL})$ serum concentrations. Standard curve ranges (ELISA 0.48-100 and Magpix 0.49-2000 pg/mL). Limit of detection for ELISA $0.3 \mathrm{pg} / \mathrm{mL}$ and Magpix 0.66 $\mathrm{pg} / \mathrm{mL}$.

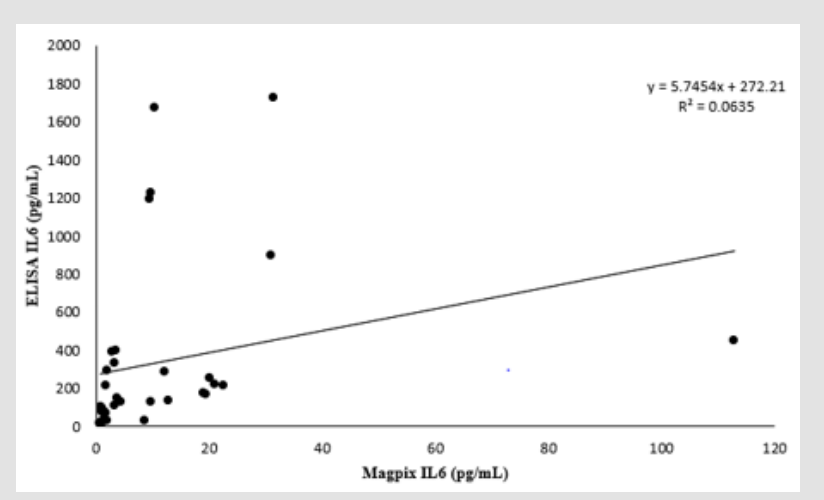

Figure 2: Method Comparison.

Note: Data expressed as ELISA IL-6 (pg/mL) vs. Magpix IL-6 (pg/mL) serum concentrations. Standard curve ranges (ELISA 1.37-1,000 and Magpix 0.18-750 pg/mL). Limit of detection for ELISA $3.0 \mathrm{pg} / \mathrm{mL}$ and Magpix 0.18 $\mathrm{pg} / \mathrm{mL}$.

\section{Discussion}

Cytokines are secreted to combat inflammation and are necessary to respond to invading pathogens. Cytokine measurement and detection in serum are essential for immune health, and the ELISA technique has long been the gold standard for analyzing cytokines in serum [14]. However, with advances in biochemical capabilities and technology, multiplex arrays offer quantitative measurement of multiple cytokines to compare to, from a single sample, unlike ELISA. The comparability has yet to be revealed for quantitative serum cytokine analyses with these two methods. Therefore, we sought to determine the differences in serum cytokine concentrations between ELISA and Multiplex techniques. The results presented in this study demonstrate the importance of understanding the limitations and discrepancies between competing analytical methods when designing, conducting, and interpreting research conclusions that involve serum cytokines. ELISA is limited to the measurement of one cytokine at a time, which restricts the ability to measure multiple biomarkers from a single biological sample $[9,19]$.

This restriction can influence an investigator's ability to answer research questions based on the sample amount available, which was the case in this observational experiment. When samples are above the cytokine range, they are typically diluted and need to be re-analyzed using another assay kit. Dilutions and remeasurements can only occur if there is sample remaining and a diluted sample may reduce the cytokine concentration and diminish any circulating inhibitors or binding protein concentrations [19]. Additionally, freeze-thaw cycles, which are common in multiple ELISAs, may also affect cytokine concentrations. Most cytokines keep their stability for up to 2 years at $-80^{\circ} \mathrm{C}$, and cytokines such as IL- $1 \beta$ and IL- 6 are degraded up to $50 \%$ within 2-3 years of storage [2]. Three freezethaw cycles are enough for most cytokines to keep stable [17] however, cytokines such as TNF- $\alpha$ may increase in concentration after each freeze-thaw period showing significant increases after three cycles [6].

ELISA techniques also lack accuracy due to insufficient blocking of immobilized antigen, specifically the hydrophobic binding of immunoglobulin in sample specimens to plastic surfaces. Research has revealed the importance of eliminating any non-specific binding in an immunological assay to avoid false positives and negative results using heterophilic blocking reagents [3,11-18]. Unfortunately, assay manufacturers do not disclose the components of their blocking agents due to proprietary information. The multiplex assay used in this study contained heterophilic blocking agents, per the manufacturer, but no further information could be given. Per our request, the manufacturers of the ELISA kits did not have any information available on their blocking agents. ELISA lacks a dynamic detection range, can be labor-intensive, expensive, and time consuming compared to Multiplex. Multiplex methodology allows for the simultaneous detection of up to 100 biomarkers from a single low volume sample, which saves time and cost.

Multiplex has the capability to perform repeated measurements of the same biomarkers from the same subjects under the same experimental assay conditions as well as the ability to reliably detect multiple biomarkers across a broad range of concentrations. Multiplex assays involve interactions between multiple cytokines in the assay solution, as a result, cross-reactivity may occur [19]. Complications may happen when rare biomarkers are assayed together. Proteins in the serum may also affect the results 
considering that all multiplex arrays occur in a solution containing freely moving molecules and antigens [19] which is an indicator that multiplex arrays are more sensitive than ELISAs to circulating proteins and inhibitors. While our research suggests that the multiplex and ELISA methods may not be comparable, additional research is warranted across a broader population due to this studies specific and small sample size with healthy and young American football players.

Athletes exhibit fewer changes in cytokine levels following a longer duration of exercise than non-athletes, suggesting that athletes have a reduced cytokine response [8]. A lack of dynamic cytokine range from our subjects is a significant limitation of our work. Additionally, more research is needed to determine the cause of these large discrepancies seen between ELISA and Multiplex methods from the exact same serum samples. The development of universal guidelines and standards to qualify an ELISA or Multiplex assay as certified for research and review by an independent organization, like the National Institute of Standards and Technology, will increase the reliability and comparability of different analytical assays conducted across multiple research outlets. A certified approval on assay kits would provide validity on research conclusions, speed up research opportunities, and increase collaboration across groups due to the validated confidence in assay performance and outcomes.

\section{Conclusion}

Navigating the research outcomes across multiple publications has become increasingly difficult for the scientific community to entrust and advance their work due to the lack of comparability across assay kits [15]. The research conclusions from this study confirm those concerns. ELISA has been described as the gold standard for serum cytokine analysis, as well as several other biomarkers. However, the findings from this study advocate further research to determine the source of discrepancies between assay techniques, there is a need for transparency on the types of blocking agents used, as well as the development of validation guidelines which would allow researchers the ability to compare results across multiple techniques and assay manufacturers. The creation of standardized assay guidelines would enhance the interpretation and translation of biomarker research.

Limitations of this study include small sample size, healthy and unique subject population, lack of manufacturer provided controls for the ELISA assays, lack of reference samples analyzed across kits, and the inability to match blocking agents across techniques. Conflict of Interest: This study was funded by and the authors are employed by the Gatorade Sports Science Institute, a division of PepsiCo, Inc. The views expressed in this manuscript are those of the authors and do not necessarily reflect the position or policy of PepsiCo, Inc.

\section{References}

1. Bernecker C, Scherr J, Schinner S, Braun S, Scherbaum WA, et al. (2013) Evidence for an exercise induced increase of TNF- $\alpha$ and IL- 6 in marathon runners. Scandanavian Journal of Medicine and Science in Sports 23(2): 207-214.

2. de Jager W, Bourcier K, Rijkers GT, Prakken BJ, Seyfert-Margolis V (2009) Prerequisites for cytokine measurements in clinical trials with multiplex immunoassays. BMC Immunology 10(52).

3. DeForge LE, Loyet KM, Delarosa D, Michael J Townsend, Jianyong Wang, et al. (2010) Evaluation of heterophilic antibody blocking agents in reducing false positive interference in immunoassays for IL-17AA, IL17FF, and IL-17AF. Journal of Immunological Methods 362(1-2): 70-81.

4. duPont NC, Wang K, Wadhwa PD, Culhane JF, Nelson EL (2005) Validation and comparison of luminex multiplex cytokine analysis kits with ELISA: Determinations of a panel of nine cytokines in clinical sample culture supernatants. Journal of Reproductive Immunology 66(2): 175-191.

5. Elshal MF, McCoy JP (2006) Multiplex bead array assays: Performance evaluation and comparison of sensitivity to ELISA. Methods 38(4): 317323.

6. Flower L, Ahuja RH, Humphries SE, Mohamed-Ali V (2000) Effects of sample handling on the stability of interleukin 6, tumornecrosis factor- $\alpha$ and leptin. Cytokine 12(11): 1712-1716.

7. Friasa MA, Virzia J, Batucac J (2019) ELISA methods comparison for the detection of auto-antibodies against apolipoprotein A1. Journal of Immunological Methods 469: 33-41.

8. Gokhale R, Chandrashekara S, Vasanthakumar KC (2007) Cytokine response to strenuous exercise in athletes and non-athletes--an adaptive response. Cytokine 40(2): 123-127.

9. Leng SX, McElhaney JE, Walston JD, Xie D, Fedarko NS, et al. (2008) ELISA and multiplex technologies for cytokine measurement in inflammation and aging research. The journals of gerontology. Series A, Biological sciences and medical sciences 63(8): 879-884.

10. Martins TB, Pasi BM, Litwin CM, Hill HR (2004) Heterophile antibody interference in a multiplexed fluorescent microsphere immunoassay for quantitation of cytokines in human serum. Clinical and Diagnostic Laboratory Immunology 11(2): 325-359.

11. Mohr M, Draganidis D, Chatzinikolaou A (2016) Muscle damage, inflammatory, immune and performance responses to three football games in 1 week in competitive male players. European journal of applied physiology 116(1): 179-193.

12. Plunkett BA, Callister R, Watson TA, Garg ML (2010) Dietary antioxidant restriction affects the inflammatory response in athletes. British Journal of Nutrition 103(8).

13. Prabhakar U, Eirikis E, Reddy M, Hugh M Davis, Bruce E Miller, et al. (2004) Validation and comparative analysis of a multiplexed assayfor the simultaneous quantitative measurement of Th1/Th2cytokines in human serum and human peripheral bloodmononuclear cell culture supernatants. Journal of Immunological Methods 291(1-2): 27-38.

14. Stefura WP, Campbell JD, Douville R (2008) Ultrasensitive ELISA for measurement of human cytokine responses in primary culture. In: MG Jones, P Lympany editors. Allergy Methods and Protocols. Methods in Molecular Medicine: Humana Press pp. 107-119.

15. Terato K, Do C, Chang J, Waritani T (2016) Preventing further misuse of the ELISA technique and misinterpretation of serological antibody assay data. Vaccine 34(39): 4643-4644.

16. Terato K, Doa CT, Cutler D, Waritania T, Shionoyab H (2014) Preventing intense false positive and negative reactions attributed to the principle of ELISA to re-investigate antibody studies in autoimmune diseases. Journal of Immunological Methods 407: 15-25. 
17. Thavasu PW, Longhurst S, Joel SP, Slevin ML, Balkwill FR (1992) Measuring cytokine levels in blood Importance of anticoagulants, processing, and storage conditions Journal of Immunological Methods 153(1-2): 115-124.

18. Waritani T, Chang J, McKinney B, Terato K (2017) An ELISA protocol to improve the accuracy and reliability of serological antibody assays. MethodsX 4: 153-165.

\section{ISSN: 2574-1241}

DOI: $10.26717 /$ BJSTR.2020.32.005313

Corey T Ungaro. Biomed J Sci \& Tech Res

(C) This work is licensed under Creative

Submission Link: https://biomedres.us/submit-manuscript.php
19. Zhou K, Fragala MS, McElhaney JE, Kuchel GA (2010) Conceptual and methodological issues relevant to cytokine and inflammatory marker measurements in clinical research. Current opinion in clinical nutrition and metabolic care 13(5): 541-547.

\begin{tabular}{ll} 
BIOMEDICAL & Assets of Publishing with us \\
\hline RESEARCHES & - Global archiving of articles \\
\hline
\end{tabular}

